\title{
First operation of the superconducting Darmstadt linear electron accelerator as an energy recovery linac
}

\author{
Michaela Arnold $\odot$, Jonny Birkhan, Jonas Pforr, Norbert Pietralla@, \\ Felix Schließmann๑, and Manuel Steinhorst \\ Institut für Kernphysik, Technische Universität Darmstadt, \\ Schlossgartenstr. 9, D-64289 Darmstadt, Germany \\ Florian Hug 1 \\ Institut für Kernphysik, Johannes Gutenberg-Universität Mainz, \\ Johann-Joachim-Becher-Weg 45, D-55128 Mainz, Germany
}

(Received 4 October 2019; accepted 7 January 2020; published 6 February 2020)

\begin{abstract}
The superconducting Darmstadt linear electron accelerator (S-DALINAC) has been operated as an energy recovery linac (ERL) for the first time. The S-DALINAC is a recirculating superconducting radiofrequency (SRF) accelerator and had been upgraded with an additional recirculation beamline. It features a path length adjustment system that provides a freedom of choice of $360^{\circ}$ for the rf phase difference between the electron bunches recirculated through the new beamline and the phase of the accelerating $\mathrm{TM}_{010}$ mode of the oscillating electromagnetic field in the SRF cavities of the accelerator. A choice of around $180^{\circ}$ for this phase difference results in a deceleration of the recirculated beam and a corresponding transfer of the particles' kinetic energy back to the energy of the electromagnetic field in the cavities. The main components relevant for ERL operation are described and data of the first operation as an SRFERL are presented.
\end{abstract}

DOI: 10.1103/PhysRevAccelBeams.23.020101

\section{INTRODUCTION}

High-energy, high-current linear accelerators (linacs) will eventually suffer from an increasingly prohibitive power consumption for the particle acceleration when beam currents above a few $\mathrm{mA}$ and electron energies in the multi-GeV range are desired.

A possible solution to this problem is the subsequent recovery of the particles' kinetic energy before disposing of the beam at low energy in a beam dump. Recirculating the beam on a decelerating phase within the linac transfers kinetic energy back to the cavity electromagnetic field. Consequently, less radio-frequency (rf) power must be provided externally to maintain the acceleration process for freshly injected beam. The energy recovery linac (ERL) technology was first proposed in 1965 by M. Tigner [1]. Several parameters can be used to quantify the efficiency of such ERLs. One of these parameters is the beam-recovery efficiency defined by

\footnotetext{
*marnold@ikp.tu-darmstadt.de
}

Published by the American Physical Society under the terms of the Creative Commons Attribution 4.0 International license. Further distribution of this work must maintain attribution to the author(s) and the published article's title, journal citation, and DOI.

$$
\mathcal{E}_{b}=\frac{E_{b, \text { max }} I_{b, \text { dump }}-P_{b, \text { dump }}}{P_{b, \max }}
$$

where $E_{b, \max }\left(I_{b, \text { dump }}\right)$ are the beam energy (beam current) at maximum energy (at the beam dump) and where $P_{b, \max }=$ $E_{b, \max } I_{b, \max }\left(P_{b, \text { dump }}=E_{b \text {,dump }} I_{b \text {,dump }}\right)$ is the beam power at top energy (at the beam dump). The beam-recovery efficiency can only be finite if the beam energy at the dump is lower than maximum and if a finite transmission to the dump is achieved. The ideal beam-recovery efficiency of $100 \%$ is obtained by a complete deceleration of the entire beam current available at maximum energy, i.e., $I_{b \text {,dump }}=I_{b \text {, max }}$ and $E_{b \text {,dump }}=0$. In general, decelerating to zero energy is not possible, so the efficiency of an ERL is limited by the injector energy, usually chosen at a few $\mathrm{MeV}$ level to reach close to ultrarelativistic electron motion before the main linac. For optimum transmission the beam-recovery efficiency is then limited to

$$
\mathcal{E}_{b, \max }=1-\frac{E_{b, \text { dump }}}{E_{b, \text { max }}} .
$$

The beam-recovery efficiency, however, does not allow to judge directly the technological gain provided by the deceleration process due to the reduction of external $\mathrm{rf}$ power required for the machine operation. For that purpose, the rf-recovery effect 


$$
\mathcal{E}_{\mathrm{rf}}=\frac{P_{\mathrm{rf}, \mathrm{acc}}-P_{\mathrm{rf}, \mathrm{ERL}}}{P_{\mathrm{rf}, \mathrm{acc}}}
$$

is more useful, where the rf beam loading is compared in situations when the beam is either blocked externally at its maximum energy $\left(P_{\mathrm{rf}, \mathrm{acc}}\right)$ or when its energy is recovered by out-of-phase recirculation to the rf cavities $\left(P_{\text {rf,ERL }}\right)$ at the same absolute amplitude of the rf field. The optimum rf-recovery effect is obtained when the beam loading in ERL mode vanishes completely.

ERLs have successfully been operated in normalconducting [2,3] or superconducting [4,5] versions at various laboratories before. Other ERLs are currently under operation/commissioning [6,7] or construction [8,9]. An overview of past, present and future ERL projects can be found in $[10,11]$. We have recently upgraded the superconducting Darmstadt linear electron accelerator (S-DALINAC) to a superconducting radio-frequency energy recovery linac (SRF-ERL). It is the first SRF-ERL operating at $3 \mathrm{GHz}$.

The correspondingly compact rf cavities used for particle acceleration comprise 20 cells. This establishes a challenging situation for ERL operation. If non-ultrarelativistic electrons $(\beta<1)$ are accelerated in a multicell structure, phase slippage, i.e., the nonoptimum phase matching of the particle bunches with the rf field for some of the cavity cells, occurs during the passage of the bunches through the cavity. Low injection energies of maximum $7.6 \mathrm{MeV}$ $(\gamma \leq 14.9, \beta \leq 0.9978)$ into the 20-cell main accelerating cavities enables the study of the phase slippage effect. This effect has also been studied theoretically as part of [12].

The S-DALINAC has operated in twice-recirculating configuration since its inauguration in 1991 [13,14]. In 2015/2016 a third recirculation beamline was installed [15], thereby allowing four acceleration passes of the linac resulting in higher final beam energy. This new beamline features a system that allows variation of the traversal path length by one full $\mathrm{rf}$ wavelength of $\lambda=10 \mathrm{~cm}$. Nominally, setting half this value will result in energy recovery.

It is the purpose of this article to present the main components of the S-DALINAC relevant for its ERL operation including the corresponding beam dynamics simulations. First evidence for its first operation as an SRF-ERL as well as different aspects of the measurements are discussed quantitatively, the value for the rf-recovery effect is reported.

\section{S-DALINAC}

The S-DALINAC is a superconducting recirculating electron accelerator. Two electron sources can be used to provide an electron beam: a thermionic gun with a preacceleration of $250 \mathrm{keV}$ and a spin-polarized source with a pre-acceleration of up to $125 \mathrm{keV}$. In the normal conducting chopper-prebuncher-section the beam is prepared for the rf acceleration with $3 \mathrm{GHz}$. A five-cell cavity is serving as

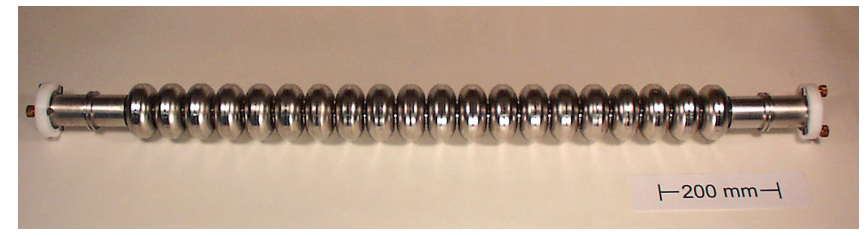

FIG. 1. Photograph of a 20-cell standard SRF cavity used at the S-DALINAC. It is made of bulk niobium and supports a $\mathrm{TM}_{010, \pi}$ resonance at $2.997 \mathrm{GHz}$.

capture section. The injector linac is completed by two 20 -cell cavities. During recirculating operation the superconducting injector linac is limited to an energy gain of up to $7.6 \mathrm{MeV}$ in order to keep the design ratio of $1: 4$ at most in between injector energy and energy gain in the main linac. The beam is then bent into the main accelerator which is capable of an energy gain of up to $30.4 \mathrm{MeV}$. It houses eight 20-cell cavities. In both the injector and linac, the cavities are $\beta=1$ with a fundamental frequency of $2.997 \mathrm{GHz}$, as shown in Fig. 1 .

Recirculating the beam up to three times increases the maximum beam energy up to $130 \mathrm{MeV}$ at beam currents of up to $20 \mu \mathrm{A}$. The floorplan of the S-DALINAC is shown in Fig. 2.

The lattice of the S-DALINAC allows different operation schemes: (i) Injector operation (ii) Single pass mode (one passage through the main linac) (iii) Once-recirculating mode (two passages through the main linac) (iv) Thricerecirculating mode (four passages through the main linac) (v) Once-recirculating ERL mode (one accelerating and one decelerating passage through the main linac) (vi) Twicerecirculating ERL mode (two accelerating and two decelerating passages through the main linac), not demonstrated yet.

The S-DALINAC supports a rich program of fundamental research in nuclear structure physics [14]. In addition, it is used for research and training in accelerator science and technology. One example is the field of energy recovery.

\section{A. Energy recovery linac mode}

The new, full path length variable beamline (up to $100 \%$ of rf wavelength) is installed in between the two pre-existing,

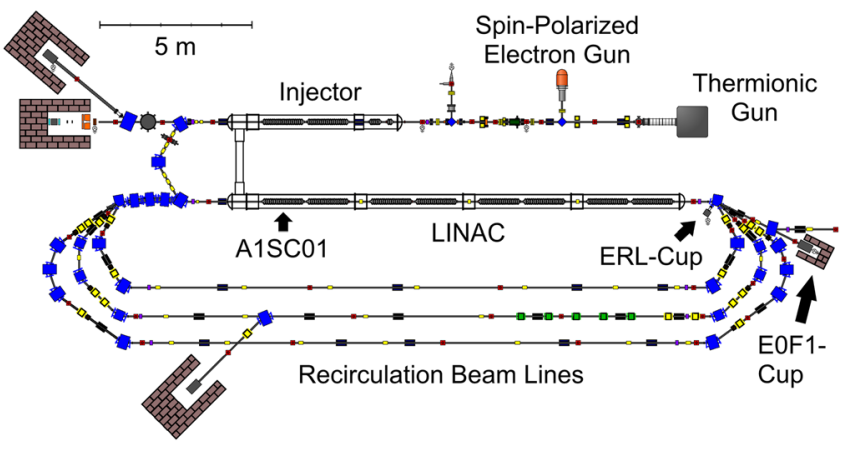

FIG. 2. Schematic floorplan of the S-DALINAC. 


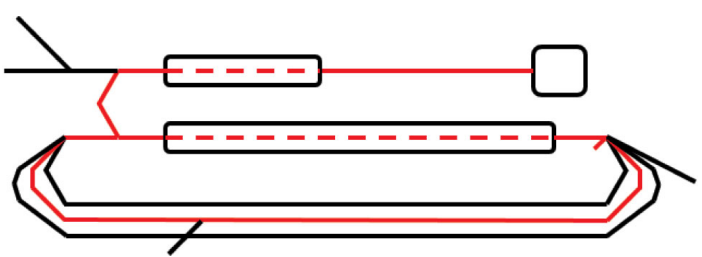

(a) Once-recirculating ERL mode.

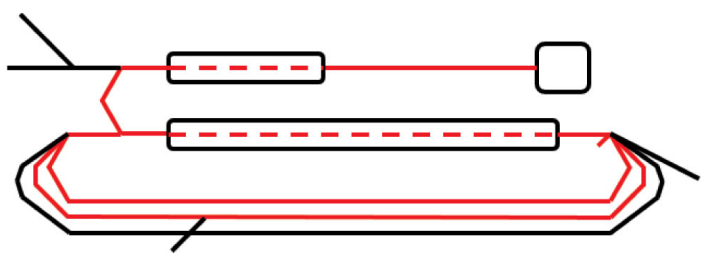

(b) Twice-recirculating ERL mode.

FIG. 3. The S-DALINAC lattice is capable of a once- or twicerecirculating ERL operation. The $180^{\circ}$ phase shift is performed in the second recirculation beamline.

limited path length variable beamlines (up to approx. $74 \%$ and $57 \%$ of full rf wavelength). This allows both once- and twice-recirculating ERL operation [16]. Figure 3 gives a schematic overview of both modes. The comparatively low beam power provided by the S-DALINAC does not exceed the rf power needed for electron acceleration. The ERL operation of the S-DALINAC does, therefore, not lead to a significant reduction of its operational costs. Instead, beam dynamical effects and the reaction of the low-level radiofrequency system for ERL operation can be studied at the S-DALINAC for basic accelerator research. In addition, junior accelerator scientists gain training and experiences in ERL operation at the S-DALINAC.

For the once-recirculating ERL the beam is preaccelerated in the injector linac. It is then bent into the main accelerator and afterwards deflected directly into the second recirculation beamline, where the phase shift of $180^{\circ}$ in comparison to the rf phase is set. Consequently, the beam reenters the main linac on the decelerating phase. After this second decelerating pass through the main linac the beam is dumped at injection energy in the ERL beam dump.

For the twice-recirculating ERL mode the preacceleration is identical. The beam is deflected initially into the first recirculation beamline after its first passage through the main accelerator. After the second acceleration it is deflected into the second recirculation beamline, which is set to $180^{\circ}$ relative phase difference. Consequently, the beam is decelerated. Then the beam retraverses the first recirculation beamline before final deceleration and dump. It should be noted that the first recirculating beamline thus carries both accelerating and decelerating beams simultaneously. This twice-recirculating ERL has not been demonstrated, yet. Results of a first partially successful test run are shown in Sec. IIID. This article presents the oncerecirculating ERL mode described above.

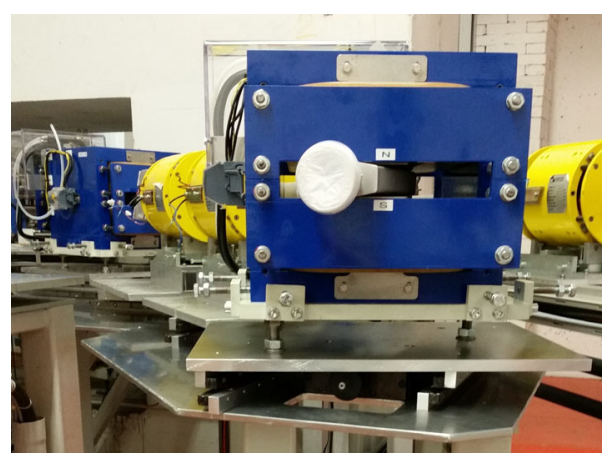

FIG. 4. Detailed view of one path length adjustment system. The magnets are installed on moveable tables sitting on ball bearing slides driven by stepper motors.

\section{B. Path length adjustment system}

The path length adjustment systems are responsible for the change in the phase of the beam. Each recirculation beamline houses such a system, consisting of dipole and quadrupole magnets on moveable tables driven by stepper motors remotely. A photograph of the arrangement of the beamline elements upon these tables in one arc of the accelerator is shown in Fig. 4.

The adjustment of the flight path through the recirculation arcs allows to adjust the point in time when a bunch is re-entering the accelerating section. In conventional accelerating operation the distance traveled by the bunches has to be a multiple of the rf wavelength of $10 \mathrm{~cm}$ (for particles traveling at the speed of light). In ERL operation an additional phase shift of half a rf wavelength is needed. Deviations from the optimum phase setting deteriorate the beam quality in both operation modes. The S-DALINAC is operated in a wide range of energies resulting in various energies for the different recirculation beamlines in the low energy regime, making a huge variation of the path lengths essential. The different strokes enable these adjustment possibilities: (i) First recirculation: up to approx. 74\% of rf wavelength (ii) Second recirculation: up to approx. 100\% of rf wavelength (iii) Third recirculation: up to approx. 57\% of $\mathrm{rf}$ wavelength

All magnets installed in the lattice have to be aligned properly. The alignment was done with a laser tracker system [17-19]. Additionally the stroke and moving axes have been measured. For the two systems of the second recirculation beamline the stroke amounts to $(50.21 \pm 0.13) \mathrm{mm}$ for the first arc and $(50.57 \pm 0.13) \mathrm{mm}$ for the second arc. A first commissioning of the system showed successful results [20].

\section{C. rf power measurement system}

The generator $\mathrm{rf}$ forward power is an observable for determining the characteristics of ERL operation. Due to the coupling between the particle beam and the rf field, beam loading occurs in the accelerating structures. This 


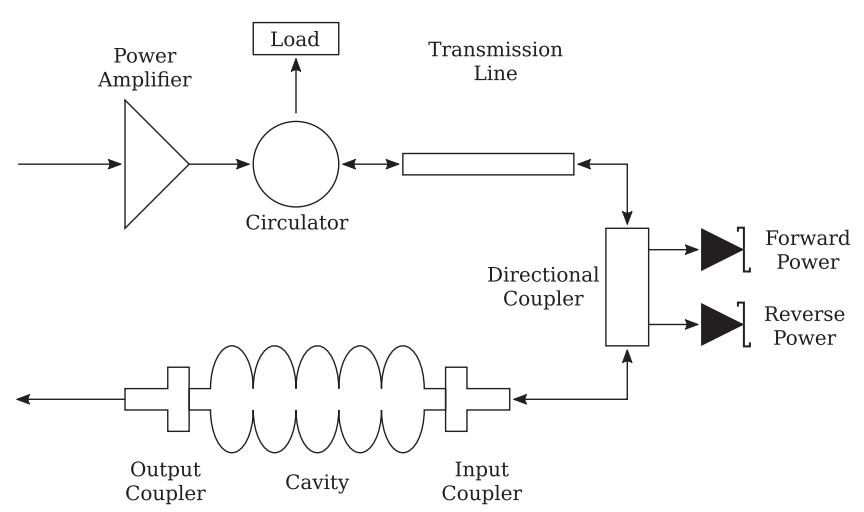

FIG. 5. Schematic of setup that was used for the rf power measurement during the ERL operation. A $40 \mathrm{~dB}$ attenuated forward and reverse if signal was extracted by coaxial directional couplers and measured by Schottky diodes.

increases forward powers depending on the power deposited in the beam. Neglecting beam losses and second order terms the forward power $P_{\mathrm{f}}$ in ERL mode can be analytically expressed by [21]

$P_{\mathrm{f}}=\frac{V_{\mathrm{acc}}^{2}}{4 R / Q Q_{L}} \cdot \frac{\beta+1}{\beta} \cdot\left[1+\left(2 Q_{\mathrm{L}} \frac{\Delta \omega}{\omega_{0}}+\frac{I_{\mathrm{b}} R / Q Q_{\mathrm{L}}}{V_{\mathrm{acc}}} \Delta \varphi\right)^{2}\right]$

The accelerating voltage is given by $V_{\text {acc }}$, the coupling factor by $\beta$, the resonance frequency by $\omega_{0}$, microphonics peak detuning by $\Delta \omega$, the beam current by $I_{\mathrm{b}}$ and the phase error of the decelerated beam due to fluctuations in the return loop path by $\Delta \varphi . R / Q$ describes the geometry of the cavity. $Q_{\mathrm{L}}$ is the loaded quality factor which quantifies the power loss per rf cycle compared to the energy stored in the resonator. The last term proportional to the beam current describes the effective beam loading in ERL operation. $\Delta \varphi$ is zero for a perfect ERL operation, corresponding to a phase shift of $180^{\circ}$, thus the beam loading term should vanish. The measurement of the effective beam loading is thus a useful observable for quantifying the rf-recovery effect.

For the power measurement $40 \mathrm{~dB}$ coaxial directional couplers in the rf transfer line in combination with Schottky diodes (model 423B from Hewlett Packard) have been used (see Fig. 5). The voltage signal measured by these Schottky diodes was read out and digitized by interface cards of the S-DALINAC control system infrastructure. The voltage signal was converted to power in units of Watt via a calibration of the Schottky diodes.

\section{Beam Dynamics Simulations}

For the upgrade with a third recirculation beamline, detailed beam dynamics simulations were performed to verify the design $[15,22,23]$. Dedicated simulations for ERL operation were also performed. The results for the oncerecirculating mode are shown in Fig. 6. Part (a) allows a
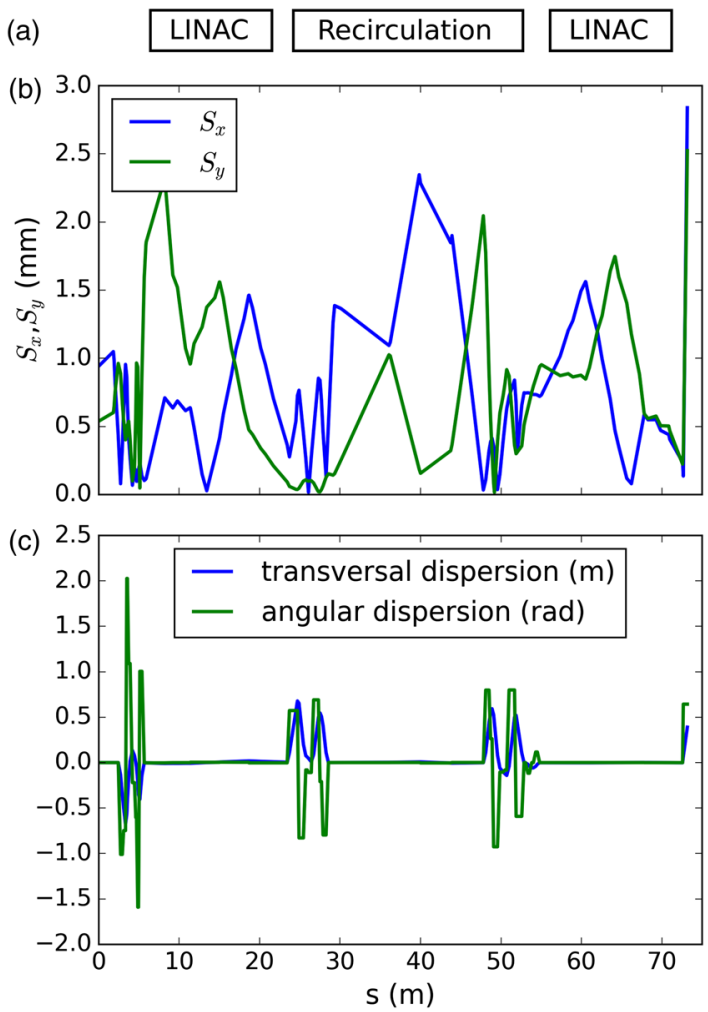

FIG. 6. Beam dynamics simulations for the once-recirculating ERL mode along the second recirculation beamline (done with ELEGANT [24]). (a) Mapping of the different sections to the simulation. (b) Beam envelope in horizontal $\left(S_{x}\right)$ and vertical $\left(S_{y}\right)$ direction. (c) Transversal and angular dispersion.

mapping of the beamline sections to the simulations shown in parts (b) and (c). The simulations include both passes of the main accelerator: one acceleration and one deceleration, as well as the traversal of the second recirculation beamline. The final section simulates to the ERL beam dump. In part (b) the beam envelopes in horizontal $\left(S_{x}\right)$ and vertical $\left(S_{y}\right)$ direction are plotted. They are within the acceptance of the machine. In part (c) the transversal and angular dispersion curves in horizontal direction are shown. They are zero in the accelerating/decelerating and the straight sections to guarantee an optimum, energy-independent transport.

\section{ENERGY RECOVERY LINAC OPERATION}

A first successful once-recirculating ERL operation of the S-DALINAC was achieved in August 2017 [25,26]. A one week period of machine development time was utilized for this purpose.

\section{A. Operational settings}

The main parameters of this run are shown in Table I. For this setting the energy gain of the injector with respect to the main linac is fixed to a ratio of $1: 8$ due to the design of the commonly used dipole magnets. The beam current used in this ERL operation is smaller than the design current for 
TABLE I. Main parameters of the once-recirculating ERL operation.

\begin{tabular}{lc}
\hline \hline Parameter & Value \\
\hline Energy gain injector (set point) & $2.5 \mathrm{MeV}$ \\
Energy gain linac (set point) & $20.0 \mathrm{MeV}$ \\
Current (before injector, set point) & $1.2 \mu \mathrm{A}$ \\
Total change in phase (set point) & $186^{\circ}$ \\
rf-recovery effect $\mathcal{E}_{\text {rf }}$ (measured) & $(90.1 \pm 0.3) \%$ \\
Beam-recovery efficiency $\mathcal{E}_{b, \max }[\mathrm{Eq} .(2)]$ & $88.9 \%$ \\
\hline \hline
\end{tabular}

conventional recirculating operation $(20 \mu \mathrm{A})$. In future ERL operations a higher beam current will be aimed at, also allowing to investigate the beam-break-up threshold current [27]. The set point of the path length adjustment system differs from the perfect ERL setting by $6^{\circ}$. This is mostly caused by strong phase slippage effects occurring at these low energies. For comparison: during a conventional once-recirculating beam time using the same energy gains of injector and main linac the first main linac cavity had to be set by approx. $15^{\circ}$ off-crest for the best beam transport. This was caused by a $\gamma \approx 5$ beam entering a 20 -cell cavity built for $\beta=1$. In case of the once-recirculating ERL operation reported here detailed studies on the phasing of the cavities have been postponed to an upcoming ERL beam time. It is most likely to have a phase slippage effect of a similar scale at the end of the deceleration, that was compensated indirectly by the set point of the path length adjustment system. The accelerating gradients of the main linac have not been set symmetrically due to operational reasons. The rf-recovery effect achieved differs from a perfect recovery mainly due to two effects: not-quantified beam losses along the recirculation beamline and a different phasing for the accelerated and decelerated beam especially in the first main accelerator cavity where the rf-recovery effect was measured.

\section{B. Power measurement}

The rf forward and reverse power of the first main accelerator cavity (A1SC01, see Fig. 2) were measured during the ERL beamtime. The forward and reverse powers are shown in the upper part of Fig. 7, with the difference shown in the lower part. This quantity corresponds to the power used by the cavity for the generation of the rf field and for acceleration.

This data was taken shortly after personnel access to the accelerator hall. This explains the drift upward of both raw traces as the rf input coupler heats up after the period of inactivity. A corresponding change of the coupling results in an additional change of the length of the self-excited loop over time. This change can in common beam operation be corrected by the operators using a loop phase shifter, when the coupler temperatures have reached equilibrium. In the presented experiments this correction has not been applied

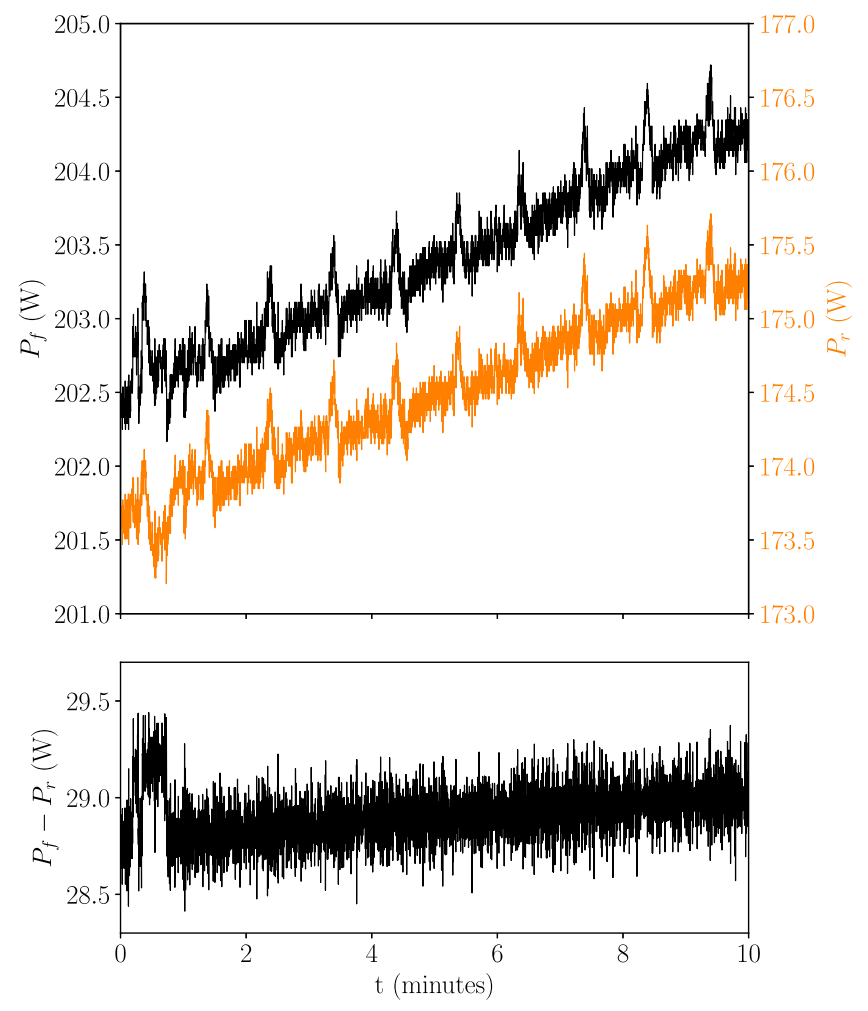

FIG. 7. Example of raw data of measured forward power $P_{\mathrm{f}}$ and reverse power $P_{\mathrm{r}}$ (top) of the first main accelerator cavity (A1SC01, see Fig. 2). The power values increase slightly over time because of heating of the input coupler due to rf losses at the coupler during the first hours of operation. The slope of the increasing powers is nearly the same recognizable in the bottom plot which shows just a small relative slope of $10^{-4} 1 / \mathrm{s}$ in the difference of both powers over time.

as temperatures were still drifting. Therefore, we need to take the drifts into account for the analysis presented in the next paragraphs. The difference of the powers over time verifies the same slope for both within an accuracy of $10^{-4}$.

The rf of the S-DALINAC is operated in cw mode such that at an equilibrium the total rf powers approach soon a constant level depending on the beam current and the loaded quality factor $Q_{\mathrm{L}}$. The important quantity for characterizing an ERL is instead the change of rf power as a function of the effective beam loading. In order to analyze the data taken during the few-hours long machine time for ERL operation quantitatively, the smooth thermal drifts of the power levels were taken into account. To correct for the linear increase of the powers over time, the powers, measured during the time without beam in the cavities, were taken as a reference. This reference time frame was used to do a linear regression. Afterwards it was subtracted from the forward and reverse powers to eliminate every effect except of the beam itself. A linear approximation could be chosen for the given analysis due to the short measurement time. The drift-corrected powers $\Delta \tilde{P}_{i}$ were calculated by 


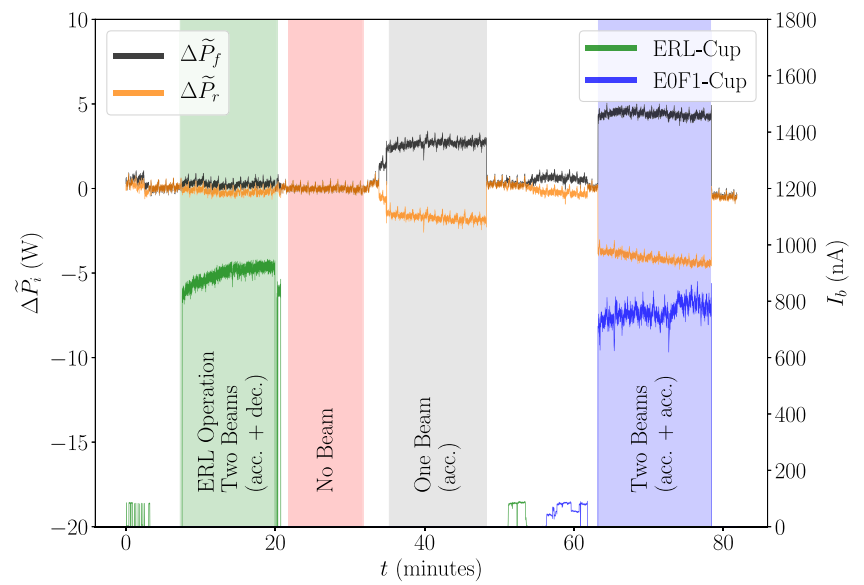

FIG. 8. During four different settings (ERL: green, no beam: red, single pass: grey, twice accelerating: blue) the changes in forward (black curve) and reverse (orange curve) rf power of the first main accelerating cavity (A1SC01, see Fig. 2) have been monitored. The beam current on the corresponding Faraday cups (ERL-Cup: green, E0F1-Cup: blue, see Fig. 2) was measured.

$$
\Delta \tilde{P}_{i}=P_{i}-\left[\left(\frac{\Delta P}{\Delta t}\right)_{i} t+\hat{P}_{0, i}\right]
$$

The indices $i$ are for forward and reverse power, the power slopes $\left(\frac{\Delta P}{\Delta t}\right)_{i}$ and the remaining offset $\hat{P}_{0, i}$ have been measured as described above.

\section{Evidence and $\mathrm{rf}$ efficiency of ERL operation}

During the ERL machine time, data on $\mathrm{rf}$ power measurements of the first main accelerating cavity as described in the preceding section, and on the beam current at the two corresponding beam dumps, were taken. Figure 8 gives an overview on the complete measurement.

The data was acquired for four different settings that refer to the following color code: (1) No beam in the main accelerator. (red) (2) Single pass: one beam is accelerated in the main accelerator. (grey) (3) Once-recirculating mode: two beams are accelerated in the main accelerator. (blue) (4) ERL mode: one beam is accelerated, another beam is decelerated in the main accelerator. (green).

The forward (black curve) and reverse power (orange curve) of the first main accelerating cavity (A1SC01) have been measured and normalized according to Eq. (5) to the time without beam but with electromagnetic field in the cavity (red band in Fig. 8). During the single pass setting (grey shading in Fig. 8) both powers changed with respect to the situation without beam due to the beam loading: The forward power increased while the reverse power dropped to a lower level. The absolute changes amount to about $2 \mathrm{~W}$ in both cases. If a second beam for acceleration is put into the cavity, the beam loading further increases (oncerecirculating mode, blue shading). Both change again by approximately the same amount. In case of the ERL operation (green shading), the effective beam loading of the cavity A1SC01 was found to almost fully cancel out. The power levels were equivalent to a beam-free linac while the beam was transported to the ERL beam dump (green curve) after having intermediately been transported through the recirculation arcs with an energy of $22.5 \mathrm{MeV}$. This is clear evidence for operation of the S-DALINAC in ERL mode.

The current measurements on the two cups is further evidence that the beam was transported to the ERL beam dump (green curve) in the ERL mode phase (green shading) as well as to the extraction beam dump (blue curve) during the once-recirculating mode (blue shading).

\section{Analytical model}

The drop in reverse power, when increasing the number of beams accelerated in the cavities, can be explained with the beam being an additional external load which couples to the electric acceleration field. The coupling coefficients $\beta_{j}$ are described by

$$
\beta_{j}=\frac{P_{j}}{P_{0}}
$$

where index $j$ includes the input coupling $\beta_{1}$, output coupling $\beta_{2}$, and beam coupling $\beta_{\mathrm{b}} . P_{0}$ is the dissipated power in the cavity and $P_{j}$ the corresponding power to the external loads. Taking the coupling into account the reflection coefficient $r$ is changing which is given by

$$
r=\frac{\beta_{1}-\left(1+\beta_{2}+\beta_{\mathrm{b}}\right)}{\beta_{1}+\left(1+\beta_{2}+\beta_{\mathrm{b}}\right)}=\sqrt{\frac{P_{\mathrm{r}}}{P_{\mathrm{f}}}} .
$$

In absence of the amplitude control of the rf control system the forward power would stay constant. In this case the power transferred to the beam would only be measurable in the reverse power. The presence of the amplitude control causes an increase in forward power to keep the accelerating field constant despite the beam loading. With higher beam coupling the reflection coefficient decreases resulting in a drop of the reverse power level. Both effects cause the steps in the corresponding powers.

In the following, an analytical model to the power data will be applied to show the expected behavior of the reverse power. In this model the drift of power is accounted in a linear change of the coupling coefficient $\beta_{1}$ and $\beta_{2}$ over time described by

$$
\beta_{k}(t)=\frac{\Delta \beta_{k}}{\Delta t} t+\beta_{k, 0}
$$

with $\frac{\Delta \beta_{k}}{\Delta}$ as the corresponding slope and $\beta_{k, 0}$ as the offset fit to the data. The power difference is given by the different power contributions 


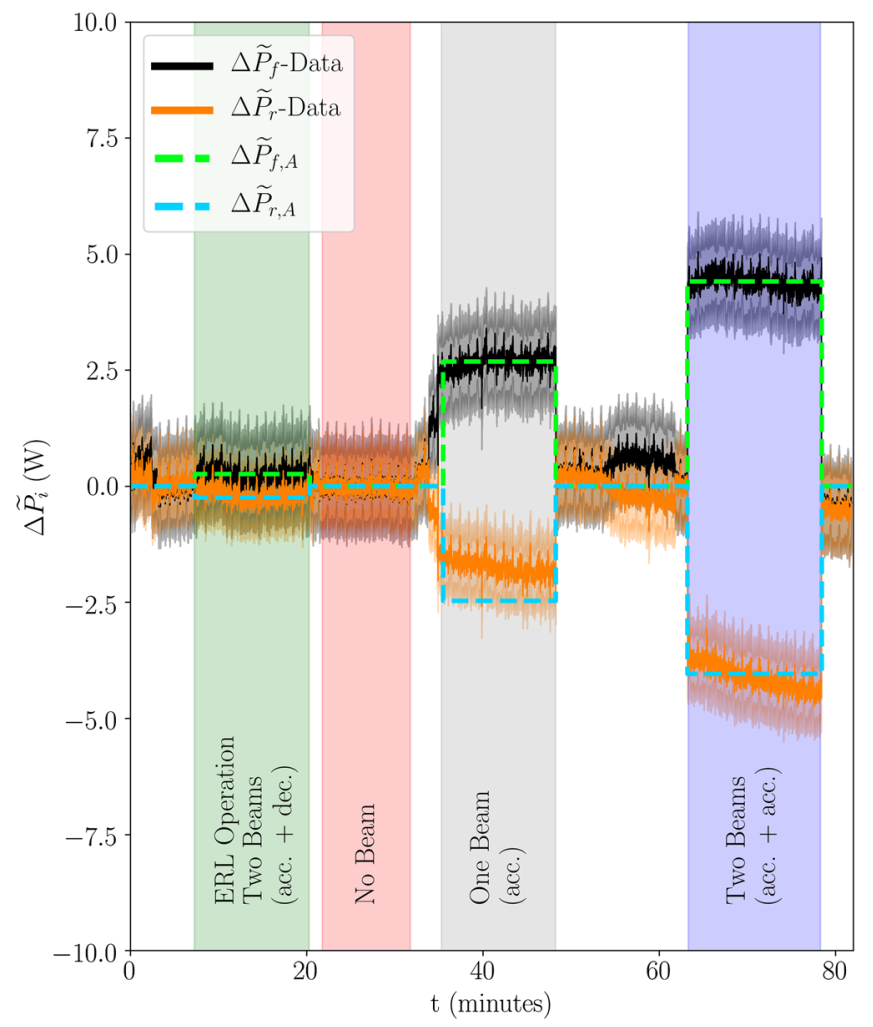

FIG. 9. Curves of the forward (black) and reverse (orange) power data including uncertainties marked as areas. The dashed lines show the curves extracted from the analytical model via curve fitting. The analytical curves show the theoretical behavior of forward (green) and reverse power (blue) in absence and presence of the beam (steps).

$$
\begin{aligned}
\left(P_{\mathrm{f}}-P_{\mathrm{r}}\right)(t) & =P_{0}+P_{2}(t)+P_{\mathrm{b}} \\
& =P_{0}\left(1+\beta_{2}(t)+\beta_{\mathrm{b}}\right)
\end{aligned}
$$

where Eq. (6) was used in the second step. Using the power difference Eq. (9) and the reflection coefficient Eq. (7) an expression for the forward power can be calculated

$$
P_{\mathrm{f}}(t)=P_{0} \frac{\left[\beta_{1}(t)+\left(1+\beta_{2}(t)+\beta_{\mathrm{b}}\right)\right]^{2}}{4 \beta_{1}(t)} .
$$

Using Eq. (7) and Eq. (10) the reverse power can be expressed as

$$
P_{\mathrm{r}}(t)=P_{0} \frac{\left[\beta_{1}(t)-\left(1+\beta_{2}(t)+\beta_{\mathrm{b}}\right)\right]^{2}}{4 \beta_{1}(t)}
$$

The beam loading in the three different situations with beam in the main linac, i.e., the coupling factors $\beta_{b \text {,ERL, }}$ $\beta_{b \text {,single }}, \beta_{b \text {,double }}$, were obtained by a fit to the data after the constants in Eq. (8) and Eq. (9) have been fixed according to the following procedure. The parameters $\frac{\Delta \beta_{2}}{\Delta t}, \beta_{2,0}$, and $P_{0}$ were obtained by a fit of Eq. (9) to the data in absence of the
TABLE II. Table of the obtained parameters in a curve fit using the analytical rf power model.

\begin{tabular}{lc}
\hline \hline Parameter & Regression result \\
\hline$P_{0}$ & $(21.743 \pm 0.001) \mathrm{W}$ \\
$\frac{\Delta \beta_{1}}{\Delta t}$ & $(5.6 \pm 0.1) \times 10^{-4} 1 / \mathrm{s}$ \\
$\beta_{1,0}$ & $33.9 \pm 0.1$ \\
$\frac{\Delta \beta_{2}}{\Delta t}$ & $(1.7 \pm 0.2) \times 10^{-5} 1 / \mathrm{s}$ \\
$\beta_{2,0}$ & $0.30 \pm 0.01$ \\
$\beta_{b, \mathrm{ERL}}$ & $0.023 \pm 0.001$ \\
$\beta_{b, \text { single }}$ & $0.238 \pm 0.001$ \\
$\beta_{b, \text { double }}$ & $0.390 \pm 0.001$ \\
\hline \hline
\end{tabular}

beam $\left(\beta_{\mathrm{b}}=0\right)$. Then, $\frac{\Delta \beta_{1}}{\Delta t}$ and $\beta_{1,0}$ were fit to the raw data using Eq. (10), in absence of the beam $\left(\beta_{\mathrm{b}}=0\right)$. Finally, the coupling factors $\beta_{b \text {,ERL }}, \beta_{b \text {,single }}, \beta_{b \text {,double }}$, were obtained by a fit of Eq. (10) to the data on the forward power in presence of beam (ERL, single linac pass, double linac pass).

Figure 9 provides a comparison of the analytical model described above with the data. Note that all model parameters were adjusted to the data on the forward power, or on the situation without beam, only. Table II lists the corresponding results of the parameters. The curve for the reverse power displayed in Fig. 9 is a prediction of the analytical model. It agrees with the data in a satisfactory way corroborating the adequacy of the modeling. Minor differences between the predicted reverse power and the data are expected to originate, e.g., from different thermal evolutions of the input and output couplers etc.

\section{2. rf-recovery effect from power measurement}

The data obtained during the power measurement shown in Fig. 8 was analyzed further to determine the rf-recovery effect. The mean values of the beam power for the different operating modes were obtained by averaging over the colored areas in Fig. 8. This data is tabulated in Table III.

The calibration curves of the Schottky diodes used for these power measurements were evaluated by a cubic spline to obtain the correct conversion from the measured voltage to the powers in Watt taking uncertainties induced by the power detector (used for calibration) and of the output of the directional coupler into account. The uncertainty of the power detector was determined to be $5 \%$ and of the directional coupler to be $2.5 \%$. The rf beam loading from

TABLE III. Mean values of the beam power with uncertainties of the distribution at the four S-DALINAC operation modes.

\begin{tabular}{lc}
\hline \hline Operation & Mean beam power $(\mathrm{W})$ \\
\hline No beam & $0.00 \pm 0.01$ \\
ERL $($ acc + dec $)$ & $0.45 \pm 0.03$ \\
One beam acc & $4.51 \pm 0.16$ \\
Two beams $($ acc + acc $)$ & $8.59 \pm 0.01$ \\
\hline \hline
\end{tabular}


Table III makes possible to quantify the rf-recovery effect of the S-DALINAC.

The rf-recovery effect $\mathcal{E}_{\text {rf }}$ [see Eq. (3)] amounts to

$$
\mathcal{E}_{\text {rf }}=(90.1 \pm 0.3) \%
$$

in the first main linac structure. This value and its uncertainty take correlations between the fit parameters (Table II) into account. The maximum achievable effect depends on the phase of the accelerated and decelerated beam with respect to the $\mathrm{rf}$ field. An effect of more than $100 \%$ is possible, if the accelerated beam is put more offcrest than the decelerated beam. A precise determination of the beam-recovery efficiency of the ERL has to take the transmission of each beam into account. It is limited to $88.9 \%$ by the injection energy, given the parameters of this ERL operation of the S-DALINAC.

During the first ERL beamtime at the S-DALINAC just one cavity could be investigated regarding the forward and reverse rf power due to the existing infrastructure. For future measurements, a power detector system was installed. It will enable us to detect the powers of all cavities simultaneously. This opens up the possibility to monitor the tuning and optimization of the phase shift achieved by path length variation in the second recirculation arcs of the S-DALINAC for further improved energy recovery performances.

\section{Uncertainty budget}

The uncertainty budget of each directly or indirectly measured quantity has been evaluated according to the GUM and GUM Supplement 1 [28,29]. Uncertainties are given in terms of the standard deviations of their corresponding quantities. The uncertainties have been propagated through the models of evaluation, including the curve fitting, through Monte Carlo calculations. Within the curve fitting of the forward and reverse power, the maximum likelihood method was applied to the posterior probability densities of each datum assuming a modified Jeffreys' prior [30] to reduce the influence of outliers.

\section{Twice-recirculating ERL test run}

At the date of submission of this paper the S-DALINAC was the only superconducting ERL world-wide that has attempted a twice-recirculating ERL operation. For this reason details on the partially successful test done in October 2018 will be given and discussed.

\section{Diagnostics}

In the twice-recirculating ERL mode the first beamline is used by a once-accelerated and once-decelerated beam at the same time. The diagnostics must be non-destructive to enable a passage of both beams at the same time. The tuning capabilities for the once-decelerated beam are limited as they always effect the once-accelerated beam and downstream sections. As diagnostics system the following devices have been used: (i) rf power measurement system to determine the beam loading in all main linac cavities to optimize the once-decelerated beam and to detect the twice-decelerated beam (ii) rf monitor system for phase measurements (one beam and sum signal of both beams) (iii) Beam loss monitor system along the first recirculation beamline and the main accelerator to optimize the beams and guide the once-decelerated beam through the first recirculation beamline (iv) $\mathrm{BeO}$ targets with a hole in the middle along the straight section of the first recirculation beamline: the once-accelerated beam passes the holes while the once-decelerated beam is visible on the outer ring (v) Checking the starting position of the path length adjustment system, that corresponds to the accelerating setting of the second beamline by the beam position and thus the correct energy in the beginning of the third recirculation beamline to define a precise $180^{\circ}$ phase shift All elements used have proven their usefulness while this test run.

\section{Overview and observations}

Due to operational constraints five of the eight main accelerator cavities have been used during this test run. The accelerating gradients have not been distributed symmetrically. The energies used for this run are listed in Table IV.

During this machine time systematic investigations on the positioning of the path length adjustment system in the second recirculation beamline and on the behavior of the once-decelerated beam in the acceptance of the first recirculation beamline have been done. Figure 10 shows an example of a typical result. The once-decelerated beam was transported on a similar orbit up to the beginning of the main linac (see F1T4, A1T1). The beam sizes on these targets and with the optical system used are overestimated using a beam current of approx. $100 \mathrm{nA}$ hitting a $\mathrm{BeO}$ target. The beam loading of the first main linac cavity showed no evidence of the second decelerated beam. During all of the different settings there have been high beam losses along the whole first recirculation beamline produced by the once-decelerated beam while the rfrecovery effect of the first recovery process could be estimated to be higher than $90 \%$ in the last main linac cavity in most of the settings without final optimization of the recovery process.

TABLE IV. Energies used for the twice-recirculating ERL test run.

\begin{tabular}{lc}
\hline \hline Parameter & Value \\
\hline Energy gain injector (set point) & $3.8 \mathrm{MeV}$ \\
Energy gain linac (set point) & $15.2 \mathrm{MeV}$ \\
Energy before deceleration (set point) & $34.2 \mathrm{MeV}$ \\
\hline \hline
\end{tabular}




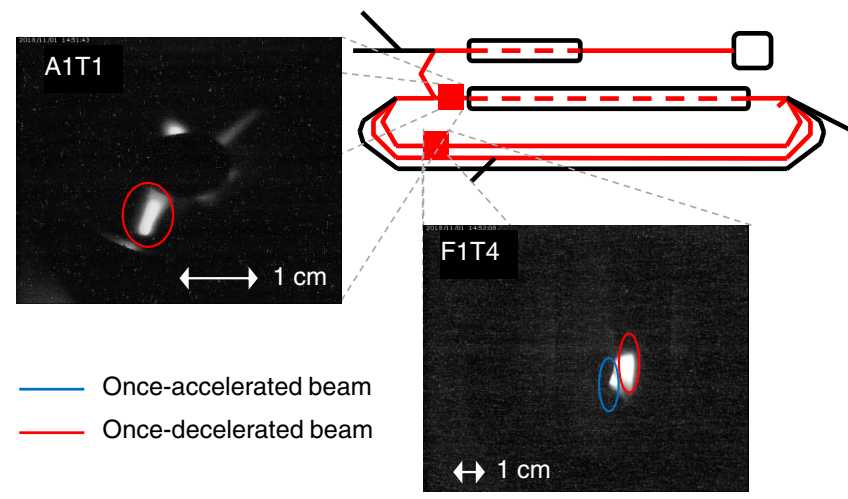

FIG. 10. BeO targets with hole show the position of the oncedecelerated beam at the end of the straight section of the first recirculation beamline ( $\mathrm{F} 1 \mathrm{~T} 4$, red circle) and in front of the main accelerator (A1T1, red circle). The once-accelerated beam is passing fully through the hole of A1T1. On F1T4 only a part of the once-accelerated beam (blue circle) is visible and not passing through the hole completely.

\section{Improvements for second test run}

We will improve the following main aspects for the second test run: (i) Optimize beam dynamics with the main goals to achieve the correct central momenta after each of the four main linac passes while minimizing the momentum spread at the same time (ii) Use the highest energy possible (limited by the cooling power of the cryoplant and the cavities) to mitigate the effects of phase slippage for the injected beam and to equalize the phase settings for the four beams in the main linac (iii) Extend the diagnostics systems: more beam loss monitors, all targets in the first recirculation beamline are changed to two targets per station ( $\mathrm{BeO}$ normal, $\mathrm{BeO}$ with hole) (iv) Use an online beam dynamics simulation to support the operators while tuning the lattice Additionally minor improvements on the lattice, stability of the beam/rf and operational procedures have been implemented meanwhile.

\section{CONCLUSION AND OUTLOOK}

The S-DALINAC is capable to be operated as once- or twice-recirculating ERL since an upgrade in 2015/2016. This mode is enabled by a path length adjustment system installed in the second recirculation beamline being capable of a total change in phase of $360^{\circ}$. In August 2017 the S-DALINAC was operated for the first time in once-recirculating ERL mode with a rf-recovery effect of $(90.1 \pm 0.3) \%$.

Up to now there is no operating multiturn SRF ERL worldwide. Concerning the twice-recirculating ERL mode at S-DALINAC, a first partially successfully test run was carried out in October 2018, but a full twice-recirculating ERL operation could not be shown yet. For the upcoming test run beam dynamics simulations are under investigation, concentrating on the effect of phase slippage and its consequences. The diagnostic systems have been further improved, facilitating an optimized measurement of two beams of the same energy traveling in the same beamline.

\section{ACKNOWLEDGMENTS}

This work was supported by the DFG through Grant No. GRK 2128.

[1] M. Tigner, A possible apparatus for electron clashing-beam experiments, Nuovo Cim. 37, 1228 (1965).

[2] G. N. Kulipanov et al., Novosibirsk free electron laserfacility description and recent experiments, IEEE Trans. Terahertz Sci. Technol. 5, 798 (2015).

[3] N. A. Vinokurov et al., Novosibirsk four-orbit ERL with three FELS, in Proceedings of IPAC 2017, Copenhagen, Denmark (JACoW, Geneva, 2017), pp. 4836-4841.

[4] F. Jackson, D. Angal-Kalinin, Y. M. Saveliev, P. H. Williams, and A. Wolski, Longitudinal transport measurements in an energy recovery accelerator with triple bend achromat arcs, Phys. Rev. Accel. Beams 19, 120701 (2016).

[5] C. Tennant, Y. Chao, D. Douglas, A. Freyberger, and M. Tiefenback, Beam characterization in the CEBFAF-ER experiment, in Proceedings of the 20th Particle Accelerator Conference, PAC-2003, Portland, OR, 2003 (IEEE, New York, 2003), pp. 3243-3245 [http://accelconf.web .cern.ch/AccelConf/p03/PAPERS/RPPG032.PDF].

[6] M. Akemoto et al., Construction and commissioning of the compact energy-recovery linac at KEK, Nucl. Instrum. Methods Phys. Res., Sect. A 877, 197 (2018).

[7] R. J. Michnoff et al., CBETA—novel supercondcuting ERL, in Proceedings of IPAC 2019, Melbourne, Australia (JACoW, Geneva, 2019), pp. 1651-1653.

[8] M. Abo-Bakr et al., Status report of the Berlin energy recovery linac project bERLinPro, in Proceedings of IPAC 2018, Vancouver, Canada (JACoW, Geneva, 2018), pp. 4127-4130.

[9] F. Hug, K. Aulenbacher, R. G. Heine, B. Ledroit, and D. Simon, MESA - an ERL project for particle physics experiments, in Proceedings of LINAC 2016, Vancouver, Canada (JACoW, Geneva, 2016), pp. 313-315.

[10] C. Tennant and D. Douglas, Overview of existing ERLs, ICFA Beam Dynamics Newsletter No. 58 (2012), p. 100, https://www-bd.fnal.gov/icfabd/Newsletter58.pdf.

[11] F. Hug, ARIES milestone report MS28 - parameter database for various ERL \& linac facilities, 2019, https://edms .cern.ch/ui/file/1817677/1.0/ARIES-Mil-MS28-Final.pdf.

[12] R. Koscica, N. Banerjee, G. H. Hoffstaetter, W. Lou, and G. Premawardhana, Energy and rf cavity phase symmetry enforcement in multiturn energy recovery linac models, Phys. Rev. Accel. Beams 22, 091602 (2019).

[13] A. Richter, Operational experience at the S-DALINAC, in Proceedings of EPAC 1996, Sitges, pp. 110-114 [http:// accelconf.web.cern.ch/AccelConf/e96/PAPERS/ORALS/ WEX02A.PDF].

[14] N. Pietralla, The institute of nuclear physics at the TU Darmstadt, Nucl. Phys. News 28, 4 (2018). 
[15] M. Arnold, C. Eschelbach, R. Grewe, F. Hug, T. Kürzeder, M. Lösler, J. Pforr, and N. Pietralla, Construction and status of the thrice recirculating S-DALINAC, in Proceedings of IPAC 2017, Copenhagen, Denmark (JACoW, Geneva, 2017), pp. 1384-1387.

[16] M. Arnold, C. Burandt, C. Eschelbach, T. Kürzeder, M. Lösler, J. Pforr, and N. Pietralla, ERL mode of S-DALINAC: Design and status, in Proceedings of ERL 2017, Geneva, Switzerland (JACoW, Geneva, 2017), pp. 40-44.

[17] M. Lösler, M. Arnold, H. Bähr, C. Eschelbach, T. Bahlo, R. Grewe, F. Hug, L. Jürgensen, P. Winkemann, and N. Pietralla, Hochpräzise Erfassung von Strahlführungselementen des Elektronenlinearbeschleunigers S-DALINAC, Fachbeitrag in zfv 140, 346 (2015).

[18] C. Eschelbach, M. Lösler, P. Winkemann, M. Arnold, and N. Pietralla, Einsatz mobiler Lasermesstechnik zur Aufnahme von Strahlführungselementen eines Elektronenlinearbeschleunigers, in Photogrammetrie-Laserscanning-Optische 3D-Messtechnik: Beiträge der 15. Oldenburger 3DTage 2016 (Tagungsband im Wichmann Verlag, Berlin and Offenbach, 2016), Vol. 1, pp. 307-318.

[19] C. Eschelbach, M. Lösler, P. Winkemann, M. Arnold, and N. Pietralla, Einsatz mobiler Lasermesstechnik bei der Erfassung von Strahlführungselementen eines Elektronenlinearbeschleunigers, in Allgemeine Vermessungs-Nachrichten (AVN), 2017, Vol. 124(3), pp. 61-69 [https://gispoint.de/artikelarchiv/avn/2017/ avn-32017/4033-einsatz-mobiler-lasermesstechnik-beider-erfassung-von-strahlfuehrungselementen-eineselektronenlinearbeschleunigers.html].

[20] M. Arnold, C. Burandt, C. Eschelbach, R. Grewe, F. Hug, M. Lösler, J. Pforr, N. Pietralla, and M. Steinhorst, First ERL operation of S-DALINAC and commissioning of path length adjustment system, in Proceedings of IPAC 2018, Vancouver, BC, Canada (JACoW, Geneva, 2018), pp. 4859-4862.

[21] Georg Hoffstaetter, Sol Gruner, and Maury Tigner, Cornell energy recovery linac: Project definition design report (PDDR), Technical report, Cornell Laboratory for
Accelerator-base Sciences and Education, June 2013, https://www.classe.cornell.edu/Research/ERL/PDDR.html.

[22] M. Arnold, F. Hug, T. Kürzeder, and N. Pietralla, Final design and status of the third recirculation for the S-DALINAC, in Proceedings of IPAC 2016, Busan, Korea (JACoW, Geneva, 2016), pp. 1717-1719.

[23] M. Arnold, F. Hug, T. Kürzeder, J. Pforr, N. Pietralla, and M. Steinhorst, Construction of a third recirculation for the S-DALINAC, in Proceedings of LINAC 2016, East-Lansing, Michigan, USA (JACoW, Geneva, 2016).

[24] M. Borland, elegant: A flexible sdds-compliant code for accelerator simulation, Advanced Photon Source Report No. LS-287, 2000.

[25] M. Arnold, J. Birkhan, F. Hug, J. Pforr, N. Pietralla, F. Schließmann, and M. Steinhorst, Once recirculating energy recovery linac operation of S-DALINAC, in Proceedings of LINAC 2018, Beijing, China (JACoW, Geneva, 2018), pp. 710-713.

[26] M. Steinhorst, M. Arnold, C. Burandt, and N. Pietralla, First energy recovery operation at the S-DALINAC: rf control stability measurements, in Proceedings of LINAC 2018, Beijing, China (JACoW, Geneva, 2018), pp. 706-709.

[27] T. Kürzeder, M. Arnold, F. Hug, L. Jürgensen, J. Pforr, and N. Pietralla, Measurements of the beam break-up threshold current at the recirculating electron accelerator S-DALINAC, in Proceedings of LINAC 2016, East Lansing, MI, USA (JACoW, Geneva, 2016), pp. 751-753.

[28] Bureau International des Poids et Mesures, Evaluation of Measurement Data-Guide to the Expression of Uncertainty in Measurement (Joint Committee for Guides in Metrology, Paris, 2008).

[29] Bureau International des Poids et Mesures, Evaluation of Measurement Data-Supplement 1 to the "Guide to the Expression of Uncertainty in Measurement" Propagation of Distributions Using a Monte Carlo Method (Joint Committee for Guides in Metrology, Paris, 2008).

[30] D. S. Sivia, Data Analysis-A Bayesian Tutorial (Oxford University Press, New York, 2006). 